\title{
The role of endoscopic ultrasound in patients with choledocholithiasis: A pilot study
}

\author{
Ahmed Alhawarey ${ }^{1 *}$, Hatem Elalfy ${ }^{1}$, Gamal Soliman ${ }^{2}$, Hussein Okasha $^{3}$, \\ Mahmoud El-bendary ${ }^{1}$ \\ ${ }^{1}$ Tropical Medicine dept., Faculty of Medicine, Mansoura Univ., Mansoura, Egypt; ${ }^{2}$ Tropical Medicine dept., \\ Faculty of Medicine, Al Azhar Univ., Cairo, Egypt; ${ }^{3}$ Internal Medicine dept., Faculty of Medicine, Cairo \\ Univ., Cairo, Egypt. \\ *ahmed.hawary1989@gmail.com
}

Received: $14-1-2020$

Accepted: $3-3-2020$

\begin{abstract}
Background and study aim. Endoscopic ultrasound (EUS) is a diagnostic modality that continues to expand its clinical applications. The aim of this study is to evaluate the diagnostic role of EUS in common bile duct stones. Patients and methods: This study was carried on 29 patients with suspected CBD stones from them only 15 patients were diagnosed as Common Bile Duct stone(s). Endoscopic retrograde cholangiopancreatography (ERCP) is considered as gold standard for diagnosis of CBD stones and compare EUS findings with that of ERCP. Results: The diagnostic performance of EUS shows that the sensitivity, specificity, PPV, NPV and accuracy of $100 \%, 92.8 \%, 93.7 \%, 100 \%$ and $96.5 \%$ respectively. It can catch 15 true positive cases with only one false positive case and 13 of 14 true negative cases. EUS is considered as a minimally invasive method with low incidence of complications, allowing exact determination of the site and size of stones. So EUS aid in better planning for CBD stone removal. Conclusion: EUS is considered as a minimally invasive method with low incidence of complications, allowing exact determination of the site and size of stones with good diagnostic performance.
\end{abstract}

Keywords: Endoscopic ultrasound, Common bile duct stones, Endoscopic retrograde cholangiopancreatography

\section{Introduction}

Choledocholithiasis occurs in $15 \%$ to $20 \%$ of patients with symptomatic cholelithiasis. The incidence of bile duct stones increases with age; up to $25 \%$ of the elderly have calculi in the bile duct at the time of cholecystectomy ${ }^{1}$. Appropriate management depends upon the identification of patients who would benefit from surgery or a therapeutic intervention. The goal of any diagnostic procedure is to confirm the presence of biliary obstruction by detecting biliary dilatation, its exact location ${ }^{2}$. A number of imaging modalities are available for the evaluation of choledocholithiasis. Current technologies include trans abdominal ultrasound (US), Computed Tomography (CT), Endoscopic Retrograde Cholangiopancreatography (ERCP), Endoscopic Ultrasound (EUS), and Magnetic Resonance Cholangiopancreatography (MR $\mathrm{CP})^{3}$. Usually abdominal Ultrasound is the initial imaging test which can demonstrate choledocholithiasis with the advantages of being noninvasive, portable, and less expensive than other imaging studies. Disadvantages include dependence on the skill of the operator for the procedure and potential technical difficulty in obese patients or patients with excessive bowel gas that overlies some organs like the pancreas and distal common bile duct ${ }^{4}$. CT scans are being used more frequently and performed rapidly, is equipped in most centers, has relatively low cost, and gives information on presence of CBD stones ${ }^{5}$. ERCP is widely used in the diagnosis and treatment of biliary and pancreatic disorders ${ }^{6}$. Getting directly into the common bile duct (CBD) is the most important step for successful procedure. The cannulation success rate depends on patient selection, the utilization of specialized catheters, and the skill and experience of the endoscopist. ERCP has long been considered the best diagnostic method for common bile duct (CBD) stones. Moreover, it allows stone removal during the same endoscopic session when combined with endoscopic sphincterotomy ${ }^{7}$. ERCP has a complication rate of $5 \%$ to $10 \%$ with a current mortality rate of less than $1 \%^{8}$. Long-term complications, such as stenosis and non-obstructive cholangitis, occur in approximately $10 \%$ of patients because of loss of biliary sphincter function. So ERCP would be reserved for patients with confirmed CBD stones as a therapeutic modality ${ }^{9}$. Endoscopic Ultrasound (EUS) is a less-invasive modality, by use of highfrequency US that is brought close to the bile duct and pancreas, and has high accuracy for diagnosing biliary stones. So, it can determine exactly the site of the biliary obstruction in relation to the ampulla of Vater ${ }^{10}$. Endoscopic ultrasound (EUS) processes high-frequency sound waves to create ultrasound images. Echoendoscopes are able to image both intramural structures and structures adjacent to the GI tract ${ }^{11}$ EUS has the advantage of visualizing the biliary tree from within the duodenum without interference from abdominal gas or fat ${ }^{12}$. MRI has been used for different diagnostic modalities in the field of hepatgastroenterology whether medical or surgical $^{13-15}$. MRCPs are performed with T2-weighted sequences that depict the biliary tract, as a highsignal intensity or bright structure without the use 
of contrast material, instrumentation or ionizing radiation. The high-signal intensity of the biliary tract noted at MRCP makes it well suited to the detection of common bile duct stones, which are seen as low-signal intensity filling defects surrounded by high-signal intensity bile ${ }^{16}$.

\section{Patients and Methods}

This study was conducted in the Tropical Medicine Department at El-Azhar University and Internal medicine department (Elebrashi Endoscopy Center) at Kasr Elaini Hospital, Cairo University during the period from June 2016 to January 2019. This study was carried on 29 patients with suspected CBD stones. Inclusion Criteria includes Elevated serum bilirubin (direct), dilated common bile duct with stone(s) which was detected by abdominal ultrasound and exclusion criteria include jaundice with normal biliary system (hepatocellular jaundice), Altered anatomy as history of gastrectomy or Rouxen-Y anastomosis, and pregnancy All the selected cases were subjected to the following: history taking, general and local examination with laboratory investigations including complete blood count liver functions, creatinine, virological markers, amylase, lipase, prothrombn time and radiological examination was done using transabdominal Ultrasound (US),

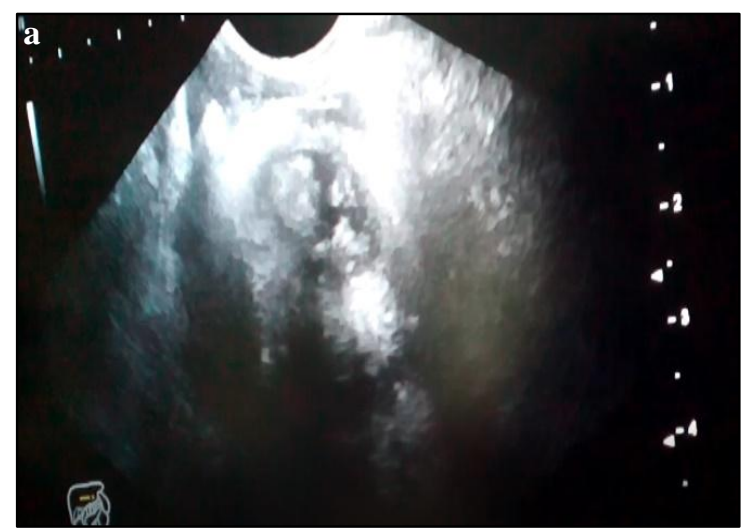

Endoscopic Ultrasound (EUS) and Endoscopic retrograde Cholangiopancreatography (ERCP). EUS examination was carried out utilizing the oblique viewing linear Pentax video machine EG-3830 UT connected to a Hitachi sonography machine EUB7000 (Tokyo, Japan) The technique was performed according to the following steps After explaining indication, possible complication of the procedure informed consent was obtained providing opportunity for questions., Connecting the patient with pulse oximetry, oxygen saturation and blood pressure measurement devices, the patient lying in left lateral position, deep sedation by Propofol 1\% IV under anesthesia specialist guidance, Tracing of CBD was done to image the entire course of the duct till its opening in the ampulla of Vater detecting any stones with characteristic posterior shadowing, fig. (1) shows the posterior shadowing of stone in Endoscopic Ultrasound.

\section{Statistical analysis}

Data are coded, processed and analyzes using SPSS version 16 (SPSS Inc., Chicago, IL). Categorical data are presented as number and percentage of total. While normally distributed continuous data are expressed as mean and standard deviation. Nonnormally distributed data are presented as median (minimum and maximum).

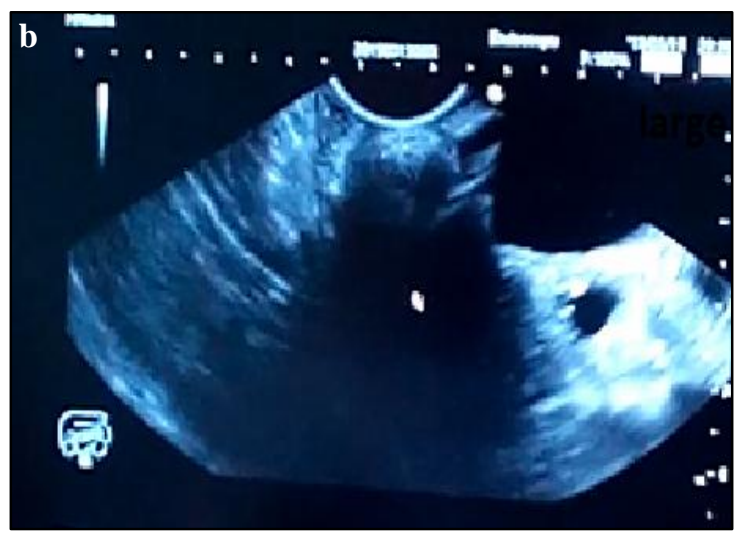

Figure 1. A case of large common bile duct stone by Endoscopic Ultrasound (EUS) a. large CBD stone, b. CBD stone with posterior shadow (arrow).

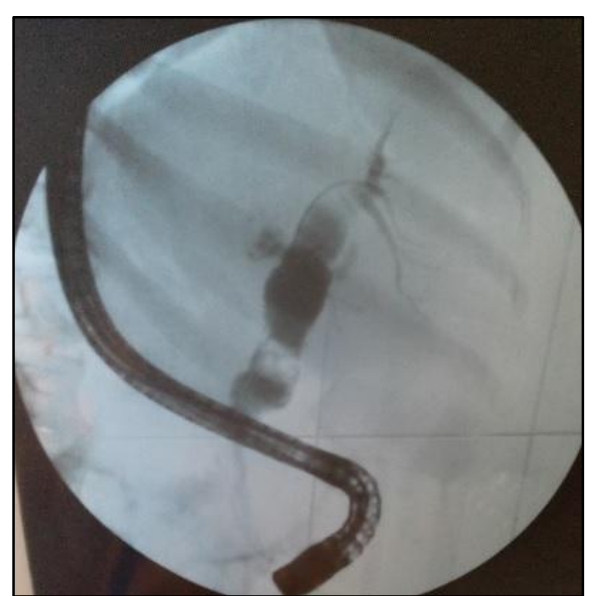

Figure 2. ERCP reveals dilated CBD with middle third stone $1.5 \mathrm{~cm}$ with dilated IHBR. 


\section{Results}

The twenty nine patients included in the study, their characteristics show that mean age was 53.2 \pm 11.6 years (male/female 42/42). Smokers were $21(25 \%)$. Mean BM was 27.48 \pm 4.81 . Frequencies and percentage of most common symptoms were abdominal pain $69(82.1 \%)$, pruritus $32(38.1 \%)$, bleeding $4(4.8 \%)$, and fever $6(7.1 \%)$. Past history of gall bladder stones was $36(42.9 \%)$, diabetes was $9(10.7 \%)$, hypertension was $6(7.1 \%)$ and cholecystectomy was $4(4.8 \%)$. The laboratory finding in the examined patients was demonstrated in tab. (1). Only 15 patients were diagnosed as Common Bile Duct stone(s). ERCP is considered as gold standard for diagnosis of CBD stones and compare EUS findings with that of ECP. Table 2 summarizes the outcome of cases in the form true positive, true negative, false positive and false negative comparing the EUS outcomes. Table 3 shows the overall accuracy, sensitivity, specificity, positive predictive value and negative predictive value for EUS.

Table 1. Laboratory finding in examined patients

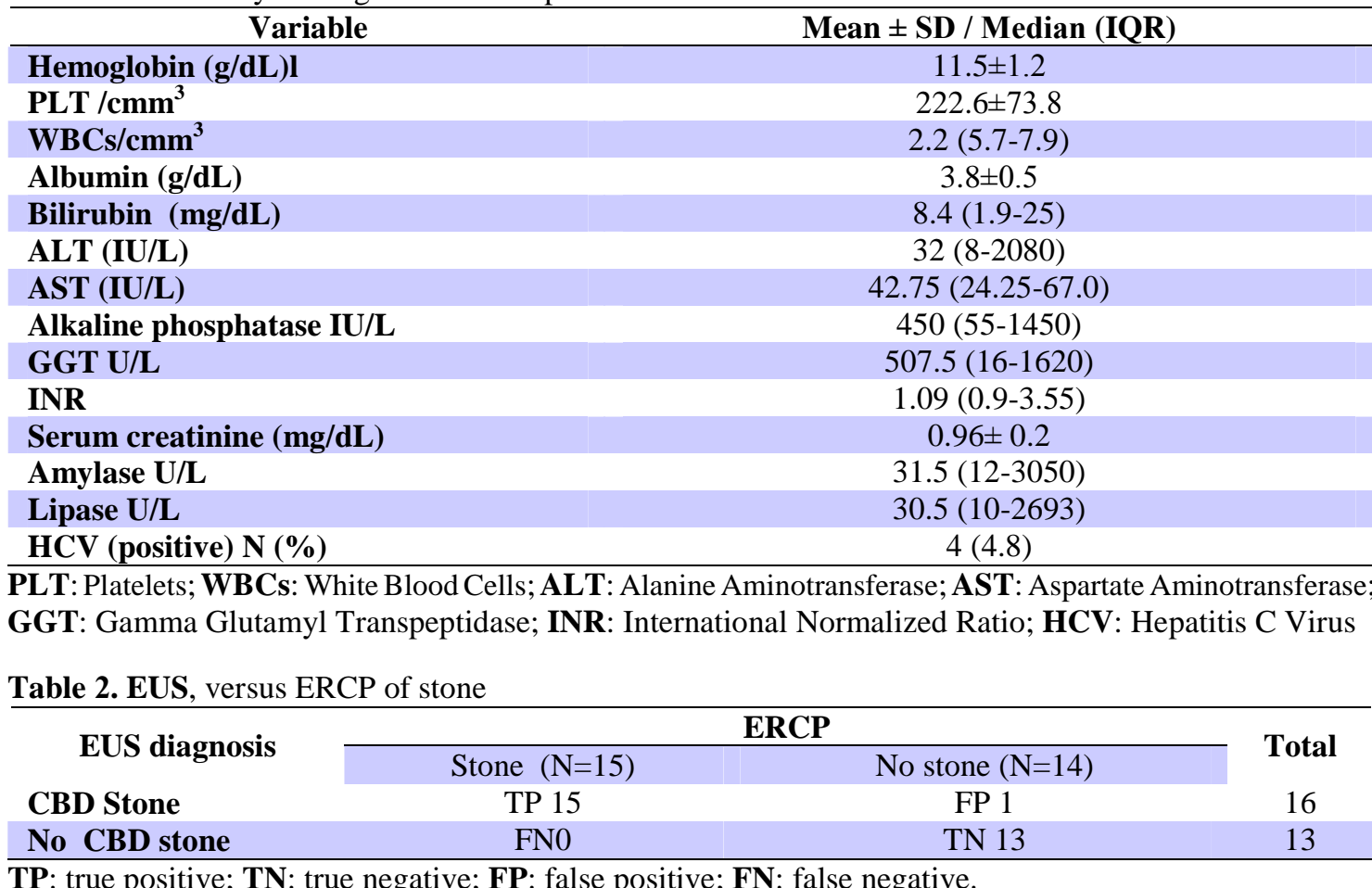

TP: true positive; TN: true negative; FP: false positive; FN: false negative.

Table 3. EUS performance in diagnosing stone.

\begin{tabular}{cccccc}
\hline & Sensitivity \% & Specificity \% & PPV\% & NPV \% & Accuracy \% \\
\hline
\end{tabular}

\section{Discussion}

Choledocholithiasis is diagnosed mainly by ERCP which considered as gold standard diagnostic tool allowing view of instrumental removal of stone(s) either by ballon or Dormia basket so this is the best reference standard using ERCP, Endoscopic sphincterotomy (ET) and instrumental exploration of the $\mathrm{CBD}^{17}$. In our study, we found that EUS as diagnostic tool shows sensitivity, specificity, PPV, NPV and accuracy of 100\%, 92.8\%, 93.7\%, 100\% and $96.5 \%$ respectively. It can catch 15 true positive cases with only one false positive case and 13 of 14 true negative cases. While a meta-analysis was done by Tse et al evaluating EUS performance in suspected choledocholithiasis has shown very good sensitivity (95\%) and specificity (98\%) for the detec- tion of CBDstones ${ }^{18}$. Aubertin et al evaluates the performance of EUS prior to laparoscopic cholecystectomy have found that the sensitivity, specificity, and positive and negative predictive values for EUS were $100 \%, 97 \%, 92 \%$, and $100 \%$, respectively ${ }^{19}$. In another study where EUS used pre-operatively; it can spare unnecessary ERCP with sensitivity of $100 \%$, Specificity $75 \%$ and NPV of $95 \%{ }^{20}$. Whereas EUS can be used before ERCP in cases with clinical suspicion of CBD stones the accuracy was $94 \%$, sensitivity of $93 \%$, specificity of $93 \%$, a positive predictive value of $98 \%$, a negative predictive value of $87 \%$. In prospective controlled study of endoscopic ultrasonography with suspected common bile duct lithiasis; the sensitivity of EUS was $93 \%$, 
specificity $97 \%$, positive predictive value $98 \%$, and negative predictive value $88 \%$ with five falsenegative cases and one false-positive ${ }^{22}$. The utilizeation of EUS before ERCP as a first-line strategy compared with ERCP alone in cases with suspected of having CED stones was associated with potential advantages in cost-effectiveness of preventing inappropriate and more invasive evaluation of the bile duct. Moreover, associated with least mean costs in EUS based strategy ${ }^{23}$. In addition to that when using EUS and ERCP during the same endoscopic session versus EUS and ERCP in two separate sessions for the management of choledocholithiasis showed that the average procedure time and days of hospitalization were significantly reduced in the first group, resulting in significant reductions in total costs. Ideally, EUS and ERCP should be combined in a single endoscopic session whenever possible to reduce risks of repeated sedation and to minimize costs ${ }^{24}$. Comparing with other diagnostic modalitiesm CT has been reported to have lower accuracy for the diagnosis of CBD stones than EUS which does not make CT the imaging technique of choice for patients with clinical suspicion of choledocholithiasis $^{\mathbf{2 5}}$. The development of multi-detector computed tomography (MDCT) has shown promise in increasing the accuracy of CT in the diagnosis of CBD stones. A study showed that MDCT was comparable with MRCP or EUS for the detection of CBD stone with $87 \%$ sensitivity, $85 \%$ specificity, and $86 \%$ accuracy $^{26}$. Nevertheless, the sensitivity of CT for detecting stones less than $5 \mathrm{~mm}$ in size remains significantly lower than those larger than $5 \mathrm{~mm}^{27}$. This differentiation of size of stones into less than $5 \mathrm{~mm}$ and more than $5 \mathrm{~mm}$ is not demonstrated in our study. In literatures; assessment of magnetic resonance cholangiopancreatography for non-invasive imaging of the biliary tree with test performance in choledocholithiasis is sensitivity, specificity, positive and negative predictive value for MRCP was $97.9 \%, 89 \%, 88.6 \%$ and $98.6 \%$ respectively ${ }^{28}$. However MRCP is a noninvasive, radiation-free imaging modality, its disadvantages include the limited spatial resolution, the difficulty of diagnosing CBD stones in the periampullary region, lack of availability in some areas, the need for operator's experience to interpret findings, and high $\operatorname{cost}^{29}$. Moreover MRCP is contraindicated in patients with metallic hardware such as pacemakers or cerebral aneurysm clips. Claustrophobic patients, estimated to represent $4 \%$ of the population often cannot complete the examination ${ }^{\mathbf{3 0}}$. There are no statistically significant differences were found between EUS and MRCP in the diagnosis of CBD stones, but a trend toward higher sensitivity and specificity for EUS compared to MRCP was evident. Nevertheless, the choice between these two techniques should depend on others factors such as resource availability, operator experience, and cost otherwise CT was inferior to them. MRCP can be utilized as an alternative when there are contraindications to sedation or when EUS is not available. EUS should be the imaging examination of choice for the detection of CBD stones, because of its high sensitivity and specificity. EUS is as effective as ERCP for detecting CBD stones, and has fewer procedural risks and complications. However, has some disadvantages, including the high cost, for expertise in the procedure, and the lack of availability in some hospitals

\section{Conclusion}

EUS has very good diagnostic performance in cases of Choledocholithiasis with sensitivity and specificity of $100 \%$ and $92.8 \%$ respectively. EUS aids in better planning for CBD stone removal so the need for ERCP only for therapeutic intervention. Moreover EUS is considered as a minimally invasive method with low incidence of complications, allowing exact determination of the site and size of stones. Nevertheless, resources, availability, operator experience, and cost limit widespread use of this modality.

\section{References}

[1] Hermann R. The spectrum of biliary stone disease. The American J. of Surgery. 1989; 158 (3): 171-173.

[2] Lillemoe K. Surgical treatment of biliary tract infections. The American Surgeon. 2000; 66 (2): 138-144.

[3] Maple J, Ben-Menachem T, Anderson, M, Appalaneni V, Banerjee S, Cash B, et al. The role of endoscopy in the evaluation of suspected choledocholithiasis. Gastrointestinal Endoscopy. 2010; 71 (1): 1-9.

[4] Pedersen O, Nordgård K, Kvinnsland S. Value of sonography in obstructive jaundice: Limitations of bile duct caliber as an index of obstruction. Scandinavian J. of Gastroenterology. 1987; 22 (8): 975-981.

[5] Kim C, Chang J, Lim Y, Kim T, Lee I, Han S. Common bile duct stones on multidetector computed tomography: attenuation patterns and detectability. World J. of Gastroenterology. 2013; WJG, 19 (11), 1788.

[6] El-Bendary M, El-Garem H, Abdel-Aziz M, Shawki S, Abdel-Razik A. Assessment of tumor markers in bile in patients with pancreaticobiliary malignancy: ERCP-based study. Afro-Egyptian J. of Infectious and Endemic Diseases. 2012; 2 (1): 7-15.

[7] Zhou P, Yao L, Xu M, Zhong Y, Gao W, He G, et al. Application of needle-knife in difficult biliary cannulation for endoscopic retrograde 
cholangiopancreatography. Hepatobiliary \& Pancreatic Diseases International. 2006; 5 (4): 590-594.

[8] Andriulli A, Loperfido S, Napolitano G, Niro G, Valvano M, Spirito F, Forlano R. Incidence rates of post-ERCP complications: A systematic survey of prospective studies. American J. of Gastroenterology. 2007; 102 (8): 1781-1788.

[9] Barthet M, Lesavre N, Desjeux A, Gasmi M, Berthezene P, Berdah S, et al. Complications of endoscopic sphincterotomy: Results from a single tertiary referral center. Endoscopy. 2002; 34 (12): 991-997.

[10] Bergman J, van Berkel M, Groen K, Schoeman N, Offerhaus J, Tytgat N, et al. Biliary manometry, bacterial characteristics, bile composition, and histologic changes fifteen to seventeen years after endoscopic sphincterotomy. Gastrointestinal endoscopy. 1997; 45 (5): 400-405.

[11] Yusuf E, Harewood C, Clain E, Levy J. International survey of knowledge of indications for EUS. Gastrointestinal endoscopy. 2006; 63 (1): 107-111.

[12] Tse F, Liu L, Barkun N, Armstrong D, Moayyedi P. EUS: A meta-analysis of test performance in suspected choledocholithiasis. Gastrointestinal Endoscopy. 2008; 67 (2): 235-244.

[13] Razek A, Massoud A, Azziz A, El-Bendary M, Zalata K, Motawea M. Prediction of esophageal varices in cirrhotic patients with apparent diffusion coefficient of the spleen. Abdominal Imaging. 2015; 40 (6): 1465-1469.

[14] Besheer T, Razek A, El Bendary M, Abd ElMaksoud M, Elalfy H, Zalata K, et al. Does steatosis affect the performance of diffusionweighted MRI values for fibrosis evaluation in patients with chronic hepatitis C genotype 4 . Turk J. Gastroenterol. 2017; 28 (4): 283-288

[15] Besheer T, Elalfy H, El-Maksoud A, ElRazek A, Taman S, Zalata K, et al. Diffusionweighted magnetic resonance imaging and micro-RNA in the diagnosis of hepatic fibrosis in chronic hepatitis C virus. World J. of Gastroenterology. 2019; 25 (11): 1366-1377

[16] Fulcher S. MRCP and ERCP in the diagnosis of common bile duct stones. Gastrointestinal Endoscopy. 2002; 56(6): S178-S182.

[17] Hawes H, Fockens P, Varadarajulu S. Endosonography E-Book. 2014; Elsevier Health Sciences.
[18] Da Costa W, Boerma D, Van Santvoort C, Horvath D, Werner J, Carter R, et al. Staged multidisciplinary step-up management for necrotizing pancreatitis. British J. of Surgery. 2014; 101 (1): e65-e79.

[19] Aubertin M, Levoir D, Bouillot L, Becheur H, Bloch F, Aouad K, et al. Endoscopic ultrasonography immediately prior to laparoscopic cholecystectomy: A prospective evaluation. Endoscopy. 1996; 28 (08): 667-673.

[20] Meroni E, Bisagni P, Bona E, Fumagalli U, Zago M, Rosati R, et al. Pre-operative endoscopic ultrasonography can optimise the management of patients undergoing laparoscopic cholecystectomy with abnormal liver function tests as the sole risk factor for choledocholithiasis: a prospective study. Digestive and Liver Disease. 2004; 36(1): 73-77.

[21] Kohut M, Nowakowska-Duława E, Marek T, Kaczor R, Nowak A. Accuracy of linear endoscopic ultrasonography in the evaluation of patients with suspected common bile duct stones. Endoscopy. 2002; 34 (04): 299-303.

[22] Prat F, Amouyal G, Amouyal P, Pelletier G, Fritsch J, Choury D, et al. Prospective controlled study of endoscopic ultrasonography and endoscopic retrograde cholangiography in patients with suspected common-bileductlithiasis. The Lancet. 1996; 347 (8994): 75-79.

[23] Buscarini E, Tansini P, Vallisa D, Zambelli A, Buscarini L. EUS for suspected choledocholithiasis: Do benefits outweigh costs? A prospective, controlled study. Gastrointestinal Endoscopy. 2003;57(4), 510-518.

[24] Fabbri C, Polifemo M, Luigiano C, Cennamo V, Fuccio L, Billi P, et al. Single session versus separate session endoscopic ultrasonography plus endoscopic retrograde cholangiography in patients with low to moderate risk for choledocholithiasis. J. of Gastroenterology and Hepatology. 2009; 24 (6): 1107-1112.

[25] Moon H, Cho D, Cha W, Cheon K, Ahn C, Kim S, et al. The detection of bile duct stones in suspected biliary pancreatitis: Comparison of MRCP, ERCP, and intraductal US. American J. of Gastroenterology. 2005; 100 (5): 1051-1057.

[26] Anderson W, Lucey C, Varghese C, Soto A. Accuracy of MDCT in the diagnosis of choledocholithiasis. American J. of Roentgenology. 2006; 187 (1): 174-180.

[27] Polkowski M, Palucki J, Regula J, Tilszer A, Butruk E. Helical computed tomographic chol- 
angiography versus endosonography for suspected bile duct stones: A prospective blinded study in non-jaundiced patients. Gut. 1999; 45 (5): 744-749.

[28] Taylor C, Little F, Hennessy F, Banting W, Smith J, Desmond V. Prospective assessment of magnetic resonance cholangiopancreatography for noninvasive imaging of the biliary tree
Gastrointestinal Endoscopy. 2002; 55 (1): 17-22.

[29] MacEneaney $P$, Mitchell T, McDermott R. Update on magnetic resonance cholangiopancreatography. Gastroenterology Clinics. 2002; 31 (3): 731-746.

[30] Kay L. Which test to replace diagnostic ERCP-MRCP or EUS? Endoscopy. 2003; 35 (05): 426-428. 